\title{
La Imprenta en la Isla Gaditana durante la Guerra de la Independencia. Libros, folletos y hojas volantes (1808-1814). Ensayo bio-bibliográfico
}

\section{Lluís Roura}

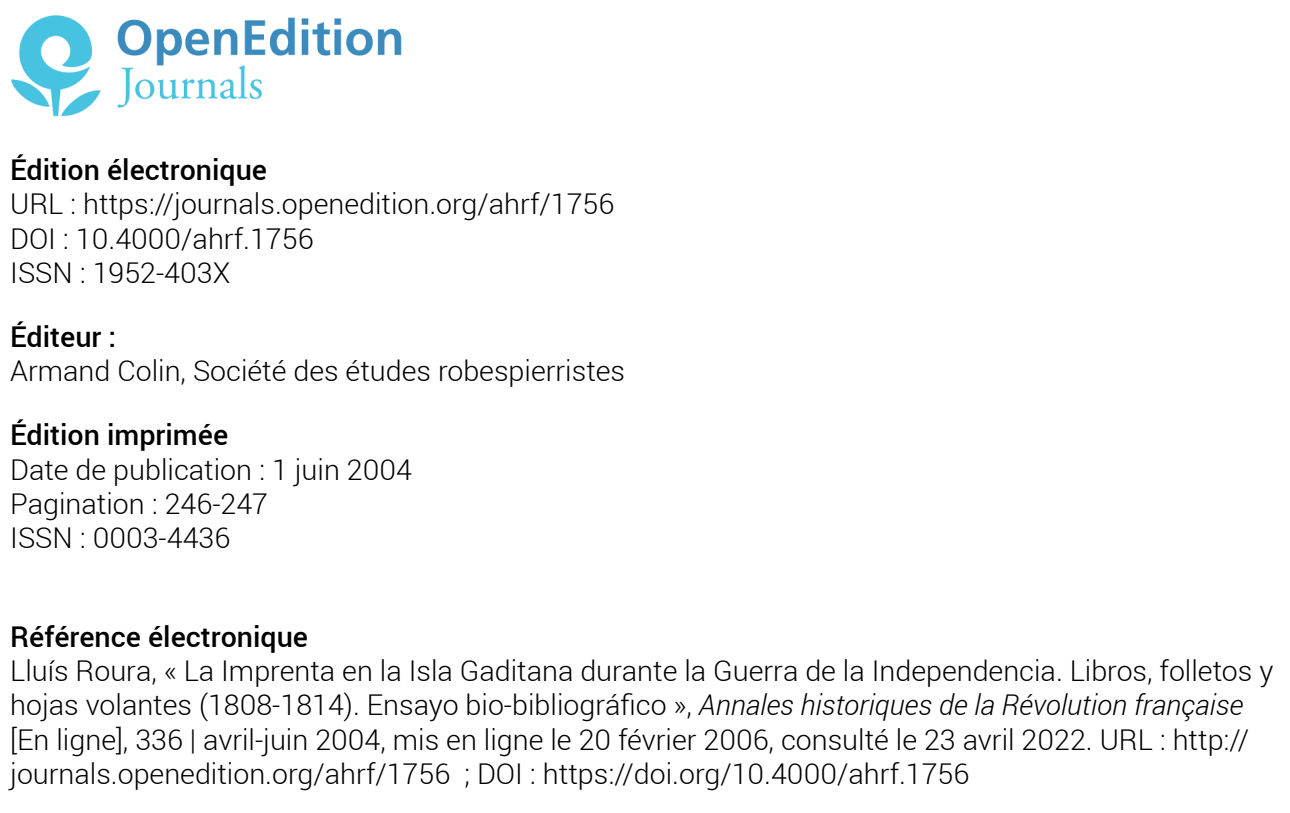

Ce document a été généré automatiquement le 23 avril 2022.

Tous droits réservés 


\title{
La Imprenta en la Isla Gaditana durante la Guerra de la Independencia. Libros, folletos y hojas volantes (1808-1814). Ensayo bio-bibliográfico
}

\author{
Lluís Roura
}

\section{RÉFÉRENCE}

Pedro Riaño de la Iglesia, La Imprenta en la Isla Gaditana durante la Guerra de la Independencia. Libros, folletos y hojas volantes (1808-1814). Ensayo bio-bibliográfico, Edición a cargo de José Manuel Fernández Tirado y Alberto Gil Novales, Madrid, Ediciones del Orto, 2004, 3 vol., 1791 p., ISBN 84-7923-296-X, $108 €$.

1 Il faut reconnaitre que les commémorations des éphémérides historiques deviennent parfois une occasion précieuse pour léguer à la postérité des apports très utiles pour le progrès de nos connaissances. C'est le cas du présent ouvrage, et ce à double raison. En 1912, Pedro Riaño de la Iglesia a présenté le manuscrit «Influencia del periodismo en la génesis, desarrollo y finalidad de la Independencia española en el período de 1808 a 1812 y otros asuntos de utilidad práctica » au concours ouvert par l'association de la presse de Cadix, à l'occasion du premier centenaire de la Guerre de l'Indépendance, et il a obtenu le prix décerné à l'issue de ce concours. Néanmoins, ce manuscrit n'a jamais été publié. Il a donc fallu la proximité du bicentenaire de l'occupation napoléonienne - bien entendu, avec le concours de la ténacité et de la passion pour l'histoire d'Alberto Gil Novales et José Manuel Fernández Tirado - pour que cette œuvre monumentale - dont le manuscrit se trouve déposé aux archives municipales de Cadix - paraisse enfin (comme le signale A. Gil Novales dans la préface, en réalité on publie ici la deuxième partie de ce qui fut une œuvre manuscrite que Riaño avait complétée, à partir de 1914, avec une 
première partie consacrée à l'étude de l'imprimerie de Cadix entre 1598 et 1808. À ce moment-là, le manuscrit de la seconde partie avait déjà le titre qui a été conservé dans l'édition actuelle).

Ces trois volumes nous offrent, avec près de 1800 pages, une étude bibliographique vraisemblablement exhaustive de l'activité de l'imprimerie à Cadix pendant les années 1808-1814. L'auteur nous présente non seulement la description bibliographique des titres recueillis - avec les rééditions, ainsi que celle des réimpressions faites à Cadix d'œuvres publiées ailleurs -, mais aussi la reproduction, in extenso ou bien des extraits les plus remarquables, d'une grande partie des publications recensées. Pedro Riaño nous livre en même temps des informations sur les prix et les tirages des imprimés, aussi bien que sur les auteurs - y compris pour les textes publiés sous des pseudonymes ou des initiales. Il s'agit toujours d'un travail de première main, réalisé principalement à partir des fonds des bibliothèques de Cadix, d'Andalousie et de Madrid, ainsi que de l'étude des journaux de l'époque.

3 L'intérêt de cette œuvre va bien au-delà de la simple érudition locale. Il est bien connu que Cadix a eu une activité d'imprimerie sans pareil en Espagne entre 1808 et 1814, et que, par ailleurs, presque tout ce qui se publiait en Espagne se trouvait ou se réimprimait à Cadix. Cette ville fut non seulement la capitale de l'Espagne durant la période d'occupation napoléonienne, mais elle fut la capitale de la Révolution libérale espagnole, et devint pour les années suivantes une référence pour la révolution en Europe et en Amérique latine. Comme le signale aussi A. Gil Novales dans la préface, l'édition de cette œuvre a le même intérêt aujourd'hui qu'elle avait en 1914 : elle représente un apport fondamental en vue de l'élaboration d'un corpus de la publicistique espagnole de la période 1808-1814 qui malheureusement nous manque encore.

Une étude très complète sur Pedro Riaño de la Iglesia (1865-1926) - avocat, historien, archiviste, bibliothécaire et poète - précède la publication de son œuvre. Dans les quelque 80 pages de son introduction, le coéditeur José Manuel Fernández Tirado nous renseigne de façon très minutieuse non seulement sur la biographie de Riaño, mais aussi sur la vie culturelle de la ville de Cadix et ses rapports avec la capitale, Madrid, particulièrement dans le contexte du premier centenaire de la Guerre de l'Indépendance. Il nous fournit aussi une description des manuscrits de Riaño, une relation de ses aléas au long de presque un siècle jusqu'à la réussite de sa publication, et une approche des critères de travail et d'organisation de son auteur. Globalement, tel que tout lecteur de cette œuvre pourra le constater, l'étude-catalogue de Riaño sur l'imprimerie à Cadix est présentée chronologiquement, année par année $(1808,1809$ et 1810 dans le premier volume de cette édition, 1811 et 1812 dans le second, enfin 1813 et 1814 dans le troisième), et pour chaque année avec l'ordre alphabétique des imprimés recensés. De plus, l'introduction nous offre aussi la recension de la cinquantaine d'œuvres et écrits de Pedro Riaño.

On doit ainsi se féliciter du travail mené par les éditeurs. Une œuvre importante, qui est un instrument fondamental pour la recherche et la documentation sur la période 1808-1814, est grâce à eux arrachée de l'oubli et mise à disposition de la communauté scientifique. Les éditeurs, en même temps, ont été à la hauteur des circonstances, n'épargnant pas les efforts pour doter ces trois volumes de trois index très complets (un index onomastique ; un autre des imprimeries et imprimeurs ; enfin, un index 
chrono-alphabétique général de tous les imprimés recueillis dans l'œuvre de Riaño) qui vont multiplier la fonction documentaire et instrumentale de l'œuvre publiée.

Il nous reste seulement à espérer que des travaux d'une envergure semblable à celui de Riaño (je pense, par exemple, à l'énorme recueil de données sur la presse espagnole pendant la Révolution libérale que, depuis plusieurs années, prépare le professeur Gil Novales) ne connaissent point aujourd'hui les problèmes subis par le manuscrit de Pedro Riaño et, donc, qu'il ne faille pas attendre le troisième centenaire pour les voir publiés... 